\title{
Soliton Domain Wall Concept: Analytical and Numerical Investigation in Digital Magnetic Recording System
}

\author{
Joseph D. Noula Tefouet, David Yemélé
}

\section{ABSTRACT}

\begin{abstract}
To introduce Soliton theory in magnetic recording systems, we begin with the profile of Domain Wall, which is key elements of recording systems, knowing that many different Domain Walls shape exist. For this, we consider the recording media as chain of atoms (spin) and, we use the Hamiltonian to describe the global state of the system; by taking into consideration interaction between the neighboring spin and anisotropic interaction. Spins are considered as classical vector; for that, we defined the cosine and sine of angles that specify the position of the spins. They are developed in Taylor's series until second order then using the approximation of continuous medium we obtained the Lagragian relation. This Lagragian enables us to describe the dynamics of spin through the wave velocity. As we are fine just the profile of domain wall it is beneficial for us to consider the wall at rest (static) and by the aid of Euler equation we obtain two simple equations; using the equilibrium conditions, the differential equation is obtained and solved by the quadratic method and separation variables method. The profile of domain wall that we obtain is at a particular position, then analytical and numerical simulation give us the opportunity to see that profile of that domain wall is a Kink, anti-Kink Soliton and also Soliton Train. Using this magnetic Soliton wave (Domain Wall), we also evaluate the playback voltage $V(x)$, the peak voltage and the half pulse width $P W_{50}$ to confirm the uses of this $\mathrm{DW}$ profile in magnetic recording systems and insure validity of this work.
\end{abstract}

Keywords: Domain Wall, Kink Soliton, Soliton Train, Output Voltage, Peak Voltage, Half Pulse Width.

\section{INTRODUCTION}

Our society is now dominated by the necessity not only to transmit information but also to store this information. The new technics of information and communication becomes inescapable in all aspect of our live. It is demonstrated many years ago that the magnetic recording systems are the best one to store and recall data. Then, it becomes important to have a good knowledge on properties of magnetic materials that made up many classes of recording system. The most important property no doubt is the one possesses by magnetic materials to orientate naturally its magnetization. This magnetic storage systems can combine parameters such as, capacity, access time, data transfer rate, and cost per bit. These key parameters define the basic performance characteristics of storage devices. From the Magnetic storage in the form of wire recording, audio recording on a wire publicized Oberlin Smith to Racetrack recording memory (Domain wall memory, DWM) publicized by Parkin in 2008, many works were done. The magnetic recorder was invented by Valdemar Poulsen. Poulsen's device recorded a signal on a wire wrapped around a drum. Although the evolution of magnetic recording system, the principle remains the same. Information is written to and read from the storage medium as the medium storage moves in front of the read-write head. The read-write head operates very close over the magnetic storage medium surface and is used to detect or modify the magnetization of the material placed immediately under it (storage medium). Magnetic surface or magnetic storage medium is conceptually divided into many small submicrometer-sized magnetic regions, referred to as magnetic domains. Each domain has a mostly uniform and nonpermanent magnetization. Note that, two consecutive magnetic regions are separated by the transition region called domain wall. In other word the region separating the consecutive domains, where the direction of magnetization changes is called magnetic domain wall (DW). Study of magnetic domain walls is remaining at the forefront of the recoding memory activity and attracts a lot of attention from engineering and physical communities. From domain walls an application point of view, their use is very promising regarding magnetic memories and many other fields of engineering [1], [2]. It is one of the driving forces of the memristor spintronics, artificial neuron network and for carrying out neuromorphic tasks [3]. Domain walls are therefore at the center of several studies and it is for this reason it has been widely studied in the literature for about thirty years [1], [4]-[7]. It should be noted that there are 
mainly two types of domain wall: The Bloch wall where the magnetization rotates in three directions of space, and the socalled Néel wall where the rotation of magnetic moments occurs in a single plane. The type of wall encountered depends both on the material used and on its structure. It depends, in particular, on the privileged direction of the magnetization. This is called an easy magnetization axis, which can have different sources, such as the crystallographic structure of the material (crystalline anisotropy), or even its shape (form anisotropy). In older Hard Disk Drive (HDD) designs, the regions were oriented horizontally and parallel to the disk surface. However, since 2005, the orientation of these regions was changed to perpendicular to allow for closer magnetic domain spacing and permit the rises of data density [8]. For reliability storage of data, the recording material needs to resist self-demagnetization, which occurs when the magnetic domains can repel each other. The older hard disk drive uses iron (III) oxide as the magnetic material, but current disks use a cobalt-based alloy [9]. This means that, during the write process a write current passes through the coil since the current needs a certain time to build up and the medium is moving under the head. This results to a magnetic transition (DW) with a finite rise time. A write head magnetizes a region by generating a strong local magnetic field and a read head detects the magnetization of the regions. Read process is occurs when the magnetization on the recording medium is detected by the MR Head and some of magnetic flux is diverted through the coil. An induced voltage is produced. The voltage produced who is dominant parameter when searching high density and is proportional to the rate of flux change. As voltage concern in recording system, the origin of this parameter can be found in electromagnetic principle in the way that: the field from a bar magnet can be represented by an equivalent current loop [10]. In the initial experiment, current flows in the head coil producing flux linkages (flux times turns) in the medium. Writing the total flux due to the longitudinal magnetization in the head coil and the head current and integrating through the volume of the medium, the total flux in the head coil can be analytically well express [11], [12]. Also, can be written in a general vector form and integral form [13], [14]. Having replay flux the readback voltage is obtained using Faraday's law and represents the signal detected by a replay head coil due to the longitudinal change in medium magnetization also called "the longitudinal replay signal" or "the replay voltage" [15]. This voltage parameter is very essential in this theory as we said early. From the beginning, HDDs used an electromagnet both to magnetize the region and read its magnetic field by using electromagnetic induction. In this type, the electric charge of electron is used to store data. Later versions of inductive heads included Metal In Gap (MIG) heads and thin film heads. As data density increased, read heads using magnetoresistance (MR) came into use, the electrical resistance of the head changes according to the strength of the magnetism from the magnetic medium. Recent development made used of spintronics; in read heads, the magnetoresistive effect was much greater than in earlier types and was dubbed "giant" magnetoresistance (GMR). Before 2008, the read and write elements are separated, but in proximity, the storage medium band moves in front of readwrite magnetic head. The read element is typically magneto- resistive while the write element is typically thin-film inductive [16]. The most one was the MRAMs nonvolatile memories exploiting the hysteretic properties of Ferromagnetic (FM) Materials or the stack of FM with heavy metal and oxides interfaces for data storage and Magnetoresistive phenomena (GMR, TMR, Anisotropic MR) for data reading. MRAMs are $2 D$ arrays of magnetic cells, so that the only way to increase density relies on the reduction of size of individual memory element [17]. The MRAM also use its spin to store data. Since 2008 with Parkin, the new type of system used domain walls as key elements. Domain wall plays an important and major role in physics of magnet in general but in the magnetic recording devices in particular. Knowing the fact that several domain wall profiles exist [18], [19], it is imperative for an optimization of the results to choose the right profile. It should be able at least to have a small width or good stability if used in racetracks. Given the zigzag domain wall being theoretically the most used, simplified modeling assuming zigzag walls can give reasonable models of transition shape as well as noise can governed the recording density [20]-[23]. For the simplicity of the problem many researchers consider that the average profile described by the zigzag wall is of a well-known shape and represented by a soliton profile. It should be noted that this soliton profile can under certain conditions give rise to soliton wave trains in the perspective of domain wall propagation for new magnetic recording systems. The functioning of Racetrack memory is by injecting pulse current in a magnetic storage media to cause the motion of domain wall. So, information can be written or read in two directions alternatively. This approach solves the problems of size of grain and develops the $3 D$ devices, this new class of memories is Racetrack (MR or DWM), in which magnetic domains are used to store information. In MR, the designs are tall columns of magnetic material arranged perpendicularly to the surface of a silicon substrate [24]. By looking and understanding how all previous systems [25] and the Racetrack Memory [26] (MR) devices work, it is important to note that the magnetic domain wall (DW) plays an important role in an old (HDD, MRAM ...) and new class (Racetrack) of magnetic recording systems. It is clear that all effort to understand, to know and apprehend the behavior of magnetic domain Walls (DW) and Magnetic domain are welcome in the progress and amelioration of performances of the magnetic recording systems, in the purpose of achievement in maximum high density digital recording systems. Despite the density of works done on this domain, it appears nevertheless that the equation of state modeling the micromagnetic dynamics of Domain Wall is commonly used in its original form. In other terms, that is, it is described by means of some quantities (Exchange interaction, anisotropic in two directions, chirality...), which could possibly limit the extent of the physical phenomena that it could present. One may wonder whether the extension of the state's equations with these parameters as three anisotropic directions can change qualitatively and quantitatively the DW behavior? The answer to this question constitutes one of the main objectives of this work. For this purpose, we will show that, from Lagrangian of the micromagnetic system and exchange interaction between nearest-neighbor through the SineGordon substrate potential, we obtain velocity of traveling 
wave, Kink or Anti-Kink solution and elliptic solution which represent Soliton train. In addition, by performing analytical calculation and numerical simulation to evaluate some important quantities in this theory which are play back voltage, peak voltage, and half pulse width. In this work, we show, prove, and obtain the DW shape (Soliton and Soliton train) using the Micromagnetic interaction in magnetic materials (recording medium), and an appropriate mathematical and analytical theories. The justification of using this DW profile is presented. This profile is used to find new analytical and numerical results concerning the magnetic digital density through the calculation of output voltage, half pulse width. All these results are compared with the old DW profile results and numerical simulation methods are used to valid the uses of this magnetic transition shape or magnetic Domain Walls (DW) for good application on magnetic recording systems.

The paper is organized as follows. In Sec.2, we describe the model of digital magnetic recording system and states equations through the Hamiltonian of that system where the Kink Soliton and the Soliton train are obtained. In Sec.3, the analytical and numerical investigation of three main parameters of this theory should be focal point of the section while Sec. 4 is consecrated to constructive discussion made to bring out the main observation of this work. Finally, Sec.5 is devoted to concluding remarks with some opening axes of futures researches work.

\section{HAMILTONIAN FORMULATION OF THE SYSTEM AND KINK-LIKE EXCITATIONS: MODEL DESCRIPTION OF DIGITAL MAGNETIC RECORDING SYSTEM AND STATE EQUATIONS}

Magnetic tape recording systems are basically constituted of four elements: the soft under layer also known as the substrate, the recording medium, the inductive write element, the GMR read sensor. The system is powered by the read and write currents and be also the source of output or replay voltage. The recording medium is characterized by the layer thickness $\delta$ and width $w$. Another important parameter of the recording systems depicted in Fig. 1 are the head-to-medium spacing $d$, the recording layer thickness $\delta$, and the domain wall width or the transition parameter $\Delta$. In general, these magnetic media appear in two types of geometric configuration depending on the orientation of the spins with regard to the direction of the thickness of the recording media and the direction of the magnetic domains with width $B$. Fig. 1(a) and (b) show a schematic representation of these two types of media.

In order to study the properties of this physics system, we will consider the magnetic storage medium as a chain of spins

with spin operator $\hat{S}$ at each site of quasi one- dimensional crystal with biaxial anisotropy and of lattice spacing $a$, that is, the magnet is assumed to have an easy axis in the $z$ direction and a hard axis in the $x$ direction (Fig. 2).

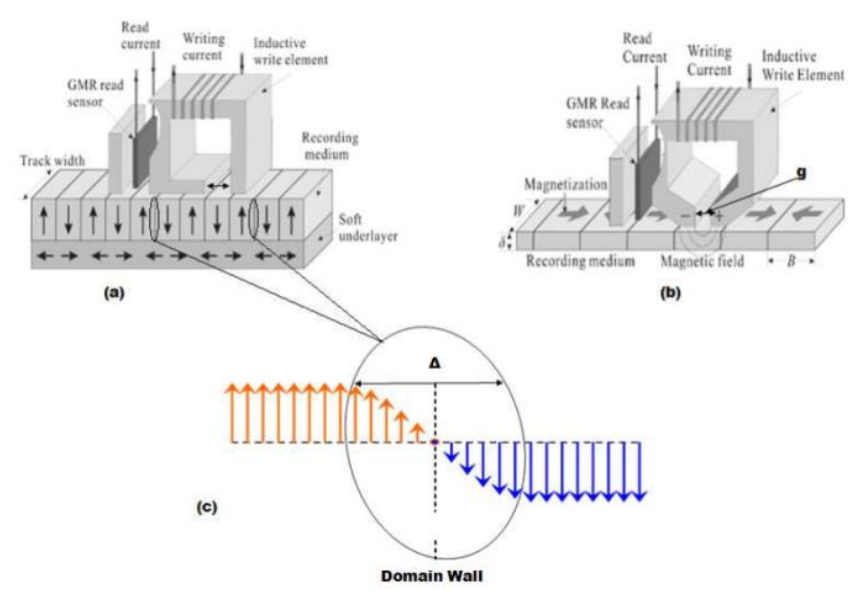

Fig. 1. (a) and (b) are representation of perpendicular and longitudinal magnetic recording head respectively with view of a ferromagnetic structure divided in two different magnetic domains, separated by magnetic Domain Wall (c) (encircledpart). (from c 2000 IBM[16]).

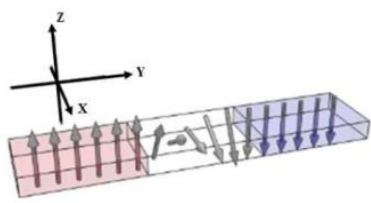

(a) Bloch Domain Wall

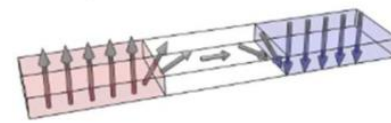

(b) Neel Domain Wall
Fig. 2. Representation of ferromagnetic structure divided in two different magnetic domains (colored zone) separated by Magnetic Domain Wall (white zone). (From Alexandre LOPEZ thesis 2006).

Thus, the model Hamiltonian can be written as:

$\hat{H}=-J \sum_{n} \hat{S}_{n} \cdot \hat{S}_{n+1}+\sum_{n}\left[K_{1}\left(\hat{S}_{n}^{z}\right)^{2}+K_{2}\left(\hat{S_{n}^{y}}\right)^{2}+K_{3}\left(\hat{S}_{n}^{x}\right)^{2}\right]+V_{p i n,}$

where the first term in the right hand side (RHS) describes the isotropic exchange interaction between nearest-neighbor pairs of spin with exchange coupling constant $J$. This parameter can be positive or negative according to whether the material is ferromagnetic or antiferromagnetic, respectively. The second term describes the magnetic anisotropic interactions with $K_{1}$ the strength of the longitudinal anisotropy and $K_{2}$ the strength of the transverse anisotropy [27].

The equation of motion for the spin operator at the $i^{\text {th }}$ site of the ferromagnet is governed by the Heisenberg principle $i \hbar \hat{S}_{i} / d t=\left[\hat{S}_{i}, \hat{H}\right]$. However, at low temperatures of the system, the classical approximation can be successfully used, that is, the giant spin operator $\hat{S}_{i}$ can be treated as a classical vector $\vec{S}_{i}$. Due to the symmetry of the problem, the vector $\vec{S}_{i}=\left(S^{x}, S^{y}, S^{z}\right)$ in the Cartesian frame is generally described by variables of spherical coordinates, namely $\theta$ and $\varphi$. Thus, one will have $\vec{S}_{i}=S\left(\sin \theta_{i} \cos \varphi_{i}, \sin \theta_{i} \sin \varphi_{i}, \cos \theta_{i}\right)$ where $S=s(s+1) \hbar$ is the magnitude of the giant spin at each site with $s$ the spin quantum number. Under this assumption, the system described by (1) possesses stable small amplitude solutions known as magnons with the dispersion relation: 


$$
\omega_{m}=\frac{J a^{3} \cos \theta_{0}}{S \hbar} k^{2}+\frac{2 a \cos \theta_{0}}{S \hbar}\left(K_{31}+K_{23} \sin ^{2} \varphi\right)
$$

or

$$
\omega_{m}=\frac{2 a K_{31}}{S \hbar}\left(1+\frac{J a^{2}}{2 K_{31}} k^{2}\right)
$$

where $\omega_{m}$ is a frequency of oscillations of an isolated giant spin around the easy axis and $k$ the wavevector. Apart from the small amplitude solutions, the system will also possess large amplitude solutions. In fact, if the domain wall is large compared to the lattice constant $a$, that is in the case of strong coupling between adjacent spins, $\vec{S}_{i}$ and $\vec{S}_{i+1}$ are very close together so that the continuum approximation can be used: $\theta_{i}(t) \rightarrow \theta(x, t)$ and $\phi_{i}=\phi(x, t)$ with $x=i a . \quad$ Similarly, $\theta_{i \pm 1}(t)$ and $\phi_{i \pm 1}(t)$ can be approximately by means of the second order Taylor expansion around $\theta_{i}(t)$ and $\phi_{i}(t)$.

Hence, the discrete Hamiltonian (1) can be replaced by a continuum form:

$$
\begin{aligned}
& H=-J S^{2}+\int \frac{d y}{a}\left\{\frac{J S^{2} a^{2}}{2}\left[\left(\frac{\partial \theta}{\partial y}\right)^{2}+\sin ^{2} \theta\left(\frac{\partial \varphi}{\partial y}\right)^{2}\right]\right. \\
& \left.+S^{2}\left[K_{1}+\left(K_{31}+K_{23} \sin ^{2} \varphi\right) \sin ^{2} \theta\right]\right\}+V_{p i n}
\end{aligned}
$$

where $K_{23}=K_{2}-K_{3}$ and $K_{31}=K_{3}-K_{1}$ are different to zero and precise the anisotropy on the longitudinal and transverse plane of the magnetic chain respectively. The pinning potential $V_{\text {pin }}$ is strong for the extrinsic defect (board, holes, scratch ...) and weak for the intrinsic defect (roughness, border between crystallites, and local fluctuation of anisotropy ...). We assume at first approximation that in this theory the media used are perfect. That is without defect and consequently $V_{\text {pin }}$ will be set to zero. Thus, the corresponding continuum Lagrangian is given by:

$$
\begin{aligned}
& L=-J S^{2}+\int \frac{d y}{a}\left\{\frac{J S^{2} a^{2}}{2}\left[\left(\frac{\partial \theta}{\partial y}\right)^{2}+\sin ^{2} \theta\left(\frac{\partial \varphi}{\partial y}\right)^{2}\right]\right. \\
& \left.+S^{2}\left[K_{1}+\left(K_{31}+K_{23} \sin ^{2} \varphi\right) \sin ^{2} \theta\right]\right\}
\end{aligned}
$$

Let us note that, the above continuum representation of the Hamiltonian and Lagrangian can be also obtained by means of the functional-integral formalism [28], [29] associated to the use of the spin coherent state [30].

For the sake of convenience, we will use the unit vector $m_{n}$ describing the direction of the spin at the given site lattice $n$, that is, $\vec{S}_{n}=S \vec{m}_{n}$ with:

$$
\vec{m}_{n}=\sin \theta_{n} \cos \varphi_{n} \vec{e}_{x}+\sin \theta_{n} \sin \varphi_{n} \vec{e}_{y}+\cos \theta_{n} \vec{e}_{z}
$$

By means of the above Lagrangian or the Landau-Lifshitz equations LLG [31], the equation describing the dynamics of the spin in the lattice is given by:

$$
\begin{aligned}
& \frac{S \hbar}{a} \sin \theta \frac{\partial \theta}{\partial t}=\frac{\delta H}{\delta \varphi} \\
& \frac{S \hbar}{a} \sin \theta \frac{\partial \varphi}{\partial t}=-\frac{\delta H}{\delta \theta}
\end{aligned}
$$

where the right-hand-side $\delta H / \delta \theta$ and $\delta H / \delta \varphi$ are functional derivatives [32], [33]. It follows two coupled equations in $\theta$ and $\varphi$ :

$$
\begin{aligned}
& \frac{S \hbar}{a} \sin \theta \frac{\partial \theta}{\partial t}=-J a^{2} \sin ^{2} \theta\left(\frac{\partial^{2} \theta}{\partial y^{2}}\right)-J a^{2} \sin 2 \theta\left(\frac{\partial \theta}{\partial y}\right)\left(\frac{\partial \varphi}{\partial y}\right) \\
& +K_{23} \sin ^{2} \theta \sin 2 \varphi
\end{aligned}
$$

and

$$
\begin{aligned}
& \frac{S \hbar}{a} \sin \theta \frac{\partial \varphi}{\partial t}=J a^{2}\left(\frac{\partial^{2} \theta}{\partial y^{2}}\right)-\frac{J a^{2}}{2} \sin 2 \theta\left(\frac{\partial \varphi}{\partial y}\right)^{2} \\
& -\sin 2 \theta\left[K_{31}+K_{23} \sin ^{2} \varphi\right]
\end{aligned}
$$

By making use of the variable $\xi=y$-vt in order to search for the solutions in the form of traveling waves at the constant velocity $v$, this set of partial differential equations lead to the following ordinary differential equations:

$$
\begin{aligned}
& -\frac{S \hbar}{a} v \sin \theta \frac{d \theta}{d \xi}=-J a^{2} \sin ^{2} \theta\left(\frac{d^{2} \varphi}{d \xi^{2}}\right)-J a^{2} \sin 2 \theta\left(\frac{d \theta}{d \xi}\right)\left(\frac{d \varphi}{d \xi}\right) \\
& +K_{23} \sin ^{2} \theta \sin 2 \varphi
\end{aligned}
$$

and

$$
\begin{aligned}
& -\frac{S \hbar}{a} v \sin \theta \frac{d \varphi}{d \xi}=J a^{2}\left(\frac{d^{2} \theta}{d \xi^{2}}\right)-\frac{J a^{2}}{2} \sin 2 \theta\left(\frac{d \varphi}{d \xi}\right)^{2} \\
& -\sin 2 \theta\left[K_{31}+K_{23} \sin ^{2} \varphi\right]
\end{aligned}
$$

describing the dynamics of the azimuthal angle $\varphi$ and orbital angle $\theta$. In general, this azimuthal angle describes the chirality of the solution. The case $\varphi=0$ has no chirality while the cases $\varphi=-\pi / 2$ and $+\pi / 2$ correspond respectively to the right-handed and left-handed solutions. This means that, in most cases, the chirality $\varphi$ is an arbitrary constant which sets the velocity of the wave. Within this condition the above two coupled (9) and (10) lead to the single equation for the variable $\theta$ :

$$
J a^{2}\left(\frac{d^{2} \theta}{d \xi^{2}}\right)-\sin 2 \theta\left[K_{31}+K_{23} \sin ^{2} \varphi\right]=0
$$

provided that the velocity takes the following explicit expression:

$$
v=\frac{a^{2} \sqrt{2 \varepsilon J K_{23}}}{S \hbar} \frac{\sin \varphi_{0} \cos \varphi_{0}}{\sqrt{1+\varepsilon \sin ^{2} \varphi}}
$$


where $\varepsilon=K_{23} / K_{31}$. Using the quadrature method, (11) leads to the following first integral:

$\frac{1}{2}\left(\frac{d \theta}{d \xi}\right)^{2}-\frac{1}{4 \Delta^{2}} V_{s}(\theta) \equiv C=-\frac{E_{d w}}{\Delta^{2}}$

where $V_{s}(\theta)=1-\cos (2 \theta)$ can be viewed as the sine-Gordon substrate potential with amplitude $V_{0}=1 / 4 \Delta^{2}$ and

$\Delta=\left(\frac{J a^{2} / 2}{K_{31}+K_{23} \sin ^{2} \theta}\right)^{1 / 2}$.

Similarly, $E_{d w}$ may be viewed as a dimensionless energy density of the total energy of the system. In this form, (13) has been extensively studied in these last decades and it appears that it exhibits many types of solutions according to the value of the parameter $C$ [34]. For localized solutions with non-vanishing boundary conditions:

$\lim _{\xi \rightarrow \infty} 2 \theta=2 \pi, \frac{d^{n} 2 \theta}{d \xi^{n}}=0$ for $2 \theta=2 \pi+2 k \pi$

with $n=1 ; 2 ; \ldots$ and $k= \pm 1 ; \pm 2 ; \ldots$, one obtains $C=0$, that is $E_{d w}=0$, and (13) admits the following well-known "kink" and "anti-kink" solution of the sine-Gordon equation [35]:

$\theta(\xi)=2 \arctan \left[\exp \left(\sigma \frac{\xi-\xi_{0}}{\Delta}\right)\right]$

This solution leads also to the well-known Walker's nonlinear localized solution given by:

$$
\cos \theta=\tanh \left(\sigma \frac{\xi-\xi_{0}}{\Delta}\right) \text { and } \sin \theta=\frac{1}{\cosh \left(\sigma \frac{\xi-\xi_{0}}{\Delta}\right)}
$$

where $\sigma= \pm 1$ is the topological charge of the kink. The case $\sigma=1$ correspond to the kink solution while the case $\sigma=$ 1stands for the anti-kink solution. Fig. 3(a) and (b) show the shapes of this solution with different polarities. This solution corresponds to a single kink solution. The linear superposition of these two kinks of different polarity constitutes a Kink-AntiKink pair separated by the length $L$ with the following analytical expression:

$\theta(\xi)=2 \arctan \left[\exp \left(+\frac{\xi-\xi_{0}}{\Delta}\right)\right]+2 \arctan \left[\exp \left(-\frac{\xi-\xi_{0}+L}{\Delta}\right)\right](18)$

Their shape is sketched in Fig. 3(c) for different values of the distance of separation $L$ which is explained as a function of the single kink width.
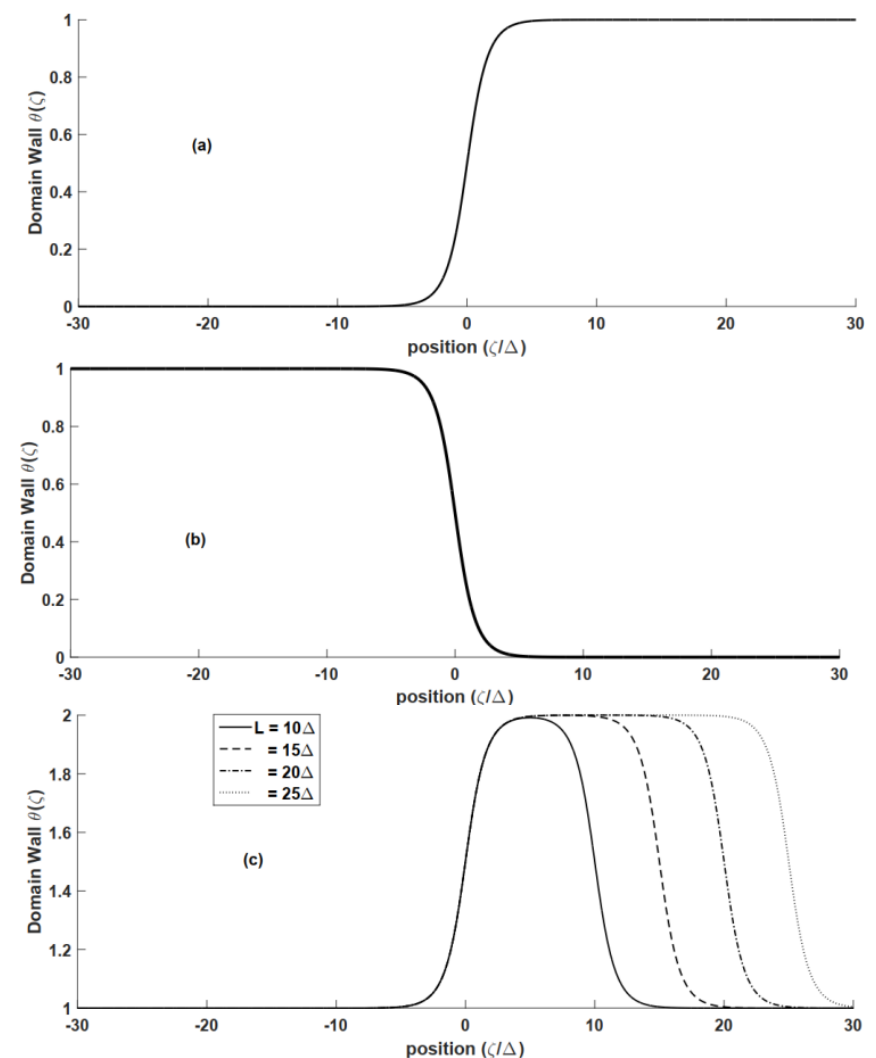

Fig. 3. Domain Wall Profile describing magnetic transition.

Apart this single kink solution, the system may also admit another type of solution when $C \neq 0$ namely $C<0$, that is $E_{d w}>0$. In fact, within this condition and after some lengthy calculations, (13) admits the following elliptic solution:

$\theta(\xi)=\arccos \left[k \times s n\left(\mp \frac{\xi-\xi_{0}}{\Delta}, k\right)\right]$

where $\operatorname{sn}(. ;$.$) is the snoidal elliptic function with modulus$ $k^{2}=1-E_{d w}$. Fig. 4 shows the shape of this solution for different values of the modulus $k$.

From the above investigations three observations can be made:

1. The solution may be viewed as a succession of kinkantikink pairs along the substrate with period $\lambda=4 \Delta K(k)$, where $K(k)$ is the complete elliptic function of the first kind.

2. A kink-antikink pair will correspond to a head to head configuration or a tail to tail configuration.

3. This periodic solution can then be used to describe the state of magnetization of the recording medium with the magnetic domain width $B$ related to this period as $B=\lambda / 2$ and the transition length described by $\Delta$. This last parameter has a great importance in digital magnetic recording system. For example, as mall value of $\Delta$ corresponds to a rapid transition, and this favors high-density recording [36]. In the following, this investigation will be used to derive some important properties of the magneto-resistive recording system namely the playback properties. 

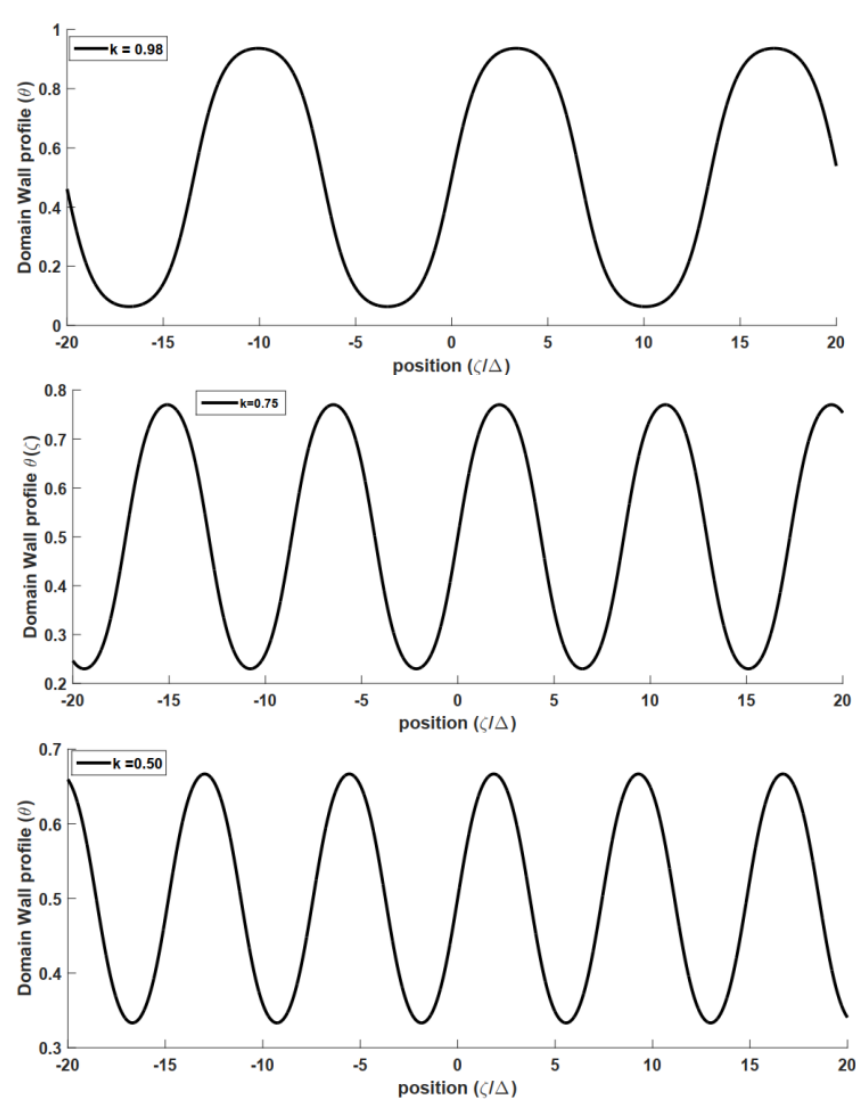

Fig. 4. Domain Wall train soliton describing a formation of more than two magnetic transition.

\section{Analytical Calculation of the Playback VolTAGE AND HALF PULSE WIDTH}

\section{A. Playback Voltage}

An important quantity characterizing the operationally of the magneto-resistive heads is the playback voltage, which is at all read and write processes. As we shall see below, the knowledge of its shape or analytical expression allows to derive other properties such as the linear density of the recording media. For the thin film media, with in-plane magnetization. As from [37]-[40], the output voltage utilizing thin film media with solely in-plane magnetization is given by:

$$
V(y)=N E v \mu_{0} M_{r} \delta \int_{-w / 2}^{w / 2} d x^{\prime} \int_{-\infty}^{+\infty} d y^{\prime} h_{y}\left(y^{\prime}+y, d\right) \frac{\partial m_{y}\left(y^{\prime}, y\right)}{\partial y^{\prime}}
$$

where $y$ is the coordinate with respect to head-medium motion of velocity $v, N$ the number of turn coil, $W$ the width track, $\delta$ the track thickness, $E$ the low frequency efficiency, $M_{r}$ the saturation remanent magnetization of the medium far from the transition and is an average of random variation of longitudinal magnetization component over the film and $m_{y}(y)$ is the longitudinal magnetization component while $h(y)$ is the longitudinal component of the head field (wide track). It is widely admitted that the magnetic field provided by the MR head, that is the record field between the pole and the keeper, can be satisfactory described by the Kalquist's approximation given by [41], [42]: $h(y)=\frac{1}{\pi g}\left\{\arctan \left(\frac{y-y_{0}+g / 2}{z}\right)+\arctan \left(-\frac{y-y_{0}-g / 2}{z}\right)\right\}$

where $z=d+g / 2$ is the distance from the lower surface of magnetoresistance element to the middle of magnetic media. In the above expression of the replay voltage (20), although the shape of the magnetization is well-known namely from different experiments works, its analytical expression remains up to now a major challenge. Thus, many expressions have been considered in the past where the most important are the arctan profile given by [43], [44]:

$m(y)=\frac{2 M_{r}}{\pi} \arctan \left(\frac{y}{a}\right)$

the hyperbolic tangent profile given by:

$m(y)=M_{r} \tanh \left(\frac{2 y}{\pi a}\right)$

and the error function expressed as:

$m(y)=\frac{2 M_{r}}{\pi} \operatorname{erf}\left(\frac{y}{a}\right)$

where $a$ is the transition length. On the basis of the investigation of the above section, for the singe pulse, we will consider the expression of the magnetization field describing the single kink given by (16). Accordingly, the magnetization field $m(y)$ will be given by:

$m_{y}\left(y^{\prime}\right)=2 M_{s} \arctan \left[\exp \left(\sigma \frac{y^{\prime}-y_{0}}{\Delta}\right)\right]$

where $\Delta$ describes the transition length as indicated above. Fig. 5 shows the shape of these last expressions.

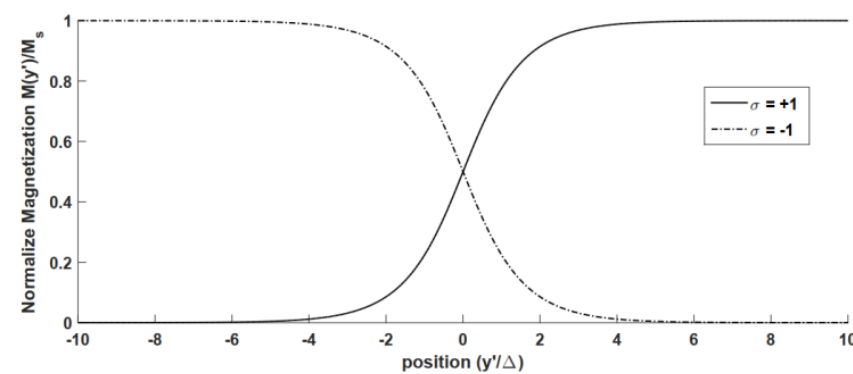

Fig. 5. Plot of magnetization against position in the media according to $\sigma$.

The analytical solution of relation (20) is considered as one of the most problem in this theory. Hence using mathematical with analytical theory and derivative of (25) into playback voltage the integral (20) representing replay voltage (output voltage) is then obtained as:

$V(y)=\frac{N E v \mu_{0} M_{r} \delta w M_{s}}{\Delta \pi g}\left(I_{1}-I_{2}\right)$ 
where $I_{1}$ and $I_{2}$ are difficult solvable integral. Finally, the replay voltage is given by:

$$
\begin{aligned}
& y \prec-\frac{g}{2} ; \frac{V(y)}{V_{0}}=-\frac{\pi}{2}\left[\arctan \frac{\cos \beta}{\sinh \left(\frac{y+g / 2}{\Delta}\right)}+\arctan \frac{\cos \beta}{\sinh \left(\frac{-y+g / 2}{\Delta}\right)}\right] \\
& -\frac{g}{2} \leq y \leq-\frac{g}{2} ; \frac{V(y)}{V_{0}}=\frac{\pi^{2}}{2}-\frac{\pi}{2}\left[\arctan \frac{\cos \beta}{\sinh \left(\frac{y+g / 2}{\Delta}\right)}+\arctan \frac{\cos \beta}{\sinh \left(\frac{-y+g / 2}{\Delta}\right)}\right] \\
& y \succ+\frac{g}{2} ; \frac{V(y)}{V_{0}}=-\frac{\pi}{2}\left[\arctan \frac{\cos \beta}{\sinh \left(\frac{y+g / 2}{\Delta}\right)}+\arctan \frac{\cos \beta}{\sinh \left(\frac{-y+g / 2}{\Delta}\right)}\right]
\end{aligned}
$$

where $V_{0}=2 N E v \mu 0 M r \delta W M s / \pi g, \beta=\mathrm{z} / \Delta$ and $z=d+\delta / 2$.

The output voltage $V(y)$ or the playback voltage of recording systems, have greatest interest because of it is at the beginning of all read-write process in high digital density recording systems, especially for magnetoresistive read-write head and Racetrack Memory. Fig. 6(a) shows the representation of the normalized output voltage $V(y)$. It is possible to compare the voltage with Domain Wall obtain in this work with other DW shape like the old Arctan and tanh profile were their output voltage are well express in [38] and [45]. With that comparison, we can see that the transition shape developed in this work has good sharp and appreciable peak voltage due to its amplitude. Moreover, Fig. 6(b) shows that at different position center of the transition medium and at each transition center, the output voltage does not change since the medium move in front of the MR Head. This indicates that, as the media move in front of the magnetic head, the voltage cannot be modified due to the media motion hence the validity of our results.
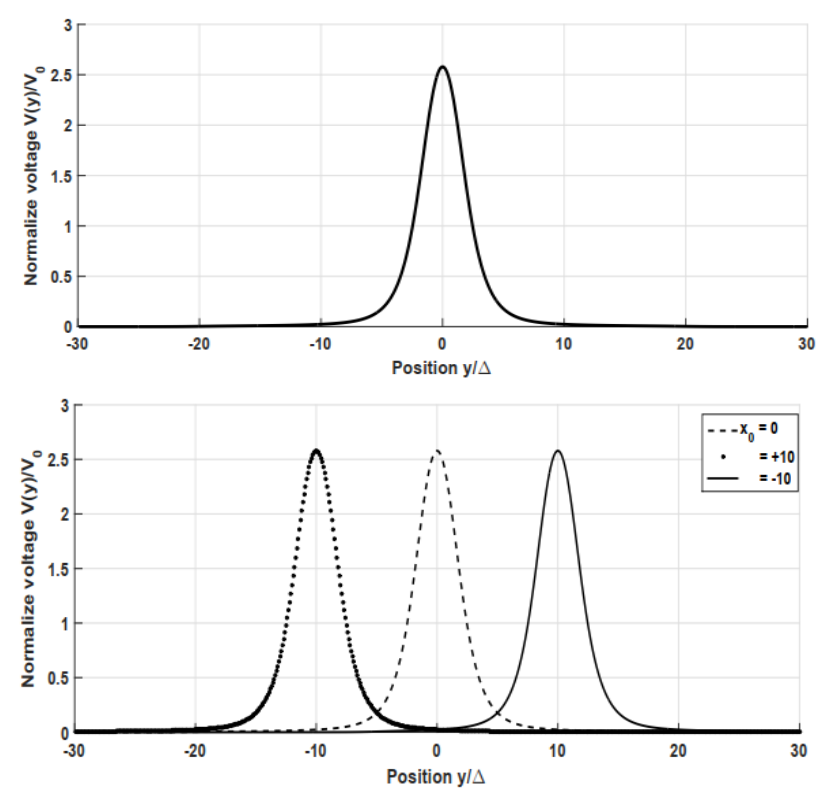

Fig. 6. Representation in (a) of the output voltage of the read-write magnetic storage systems with curve plotted at $y_{0}=0$. (b) The output voltage plotted at different transition center $y_{0}=-10 ; 0$ and +10 .

The validity of present investigation is confirmed in Fig.7 were the voltage of present DW model versus position is plotted at three different values of head-medium spacing $d$. We observe that the peak voltage increases as $d$ decreases and so leads to the decrease of half width $y_{50}$, of Bit spacing, and the increase in Linear Density or Areal Density. It should be noted that the output voltage versus position for different head-medium separation values is necessary to show the importance of putting the medium very close to the head and avoid the spreading of voltage pulse which can cause a large $y_{50}$. Note also that the proximity of medium and head can make storage medium to erode. This is another question and will not be discussed in this work.

The output voltage $V(y)$ or the playback voltage is very important on recording system not only because it is at beginning of read and writes process but adding the impact of its peak value $V_{\text {peak }}$.

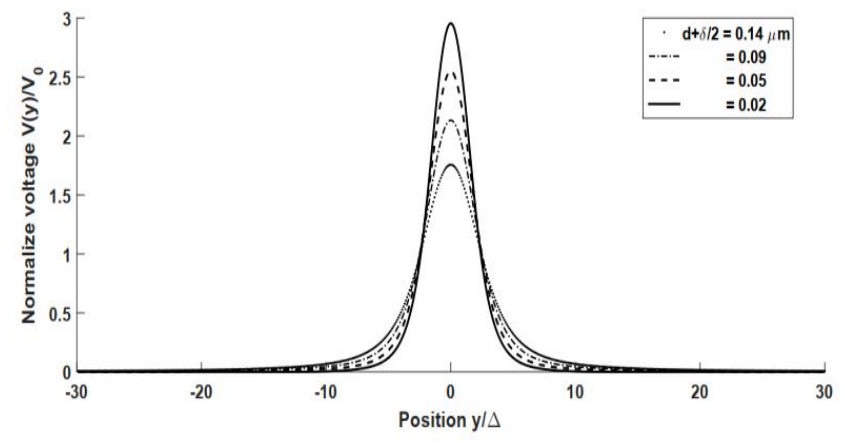

Fig. 7. Comparison graph of output voltage $V(y)$ due to the magnetic distance $d$.

By the fact that $V_{\text {peak }}$ is obtain at $y=0$, we fine this peak voltage using the voltage relation among the range $-g / 2 \leq y \leq$ $g / 2$, and the peak voltage obtain is given by:

$V_{\text {Peak }}=V_{0}\left(\frac{\pi^{2}}{2}-\pi \arctan \frac{\cos \beta}{\sinh \left(\frac{g}{2 \Delta}\right)}\right)$

This peak voltage can be used to obtain the small width at half $y_{50}$. We plot in Fig. 8 in a comparison graph peak voltage versus $g / 2 \Delta$ of two different transition profiles where the graph exhibited peak voltage for transition domain wall shape presented in this work greater than the simple arctan transition shape. This emphasize the fact that, with this $\arctan (\exp )$ shape it is possible to have good peak voltage which gives the smallest half pulse width or smallest bit length (B) and then the higher the linear or areal density.

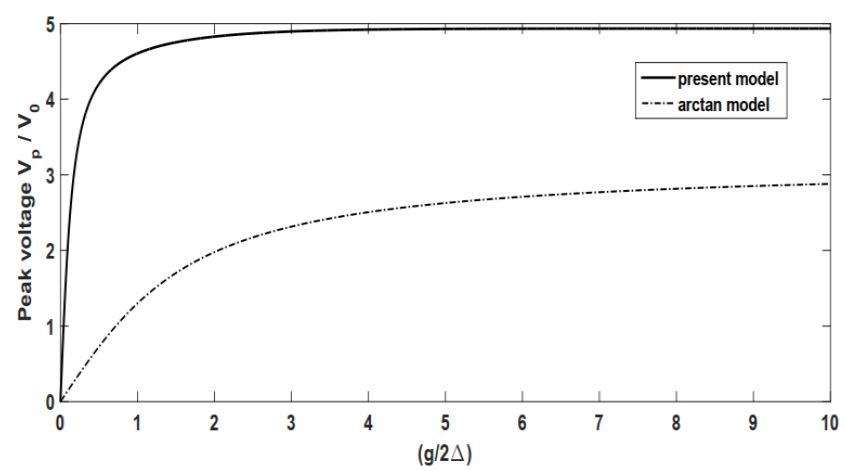

Fig. 8. Peak voltage comparison graph for two different shape, the simple $\arctan$ and the $\arctan (\mathrm{exp})$ transition shape. Here the model presents the very good peak voltage who gives right to obtain the preferable half pulse more accurate and narrower than others even that the magnetic recording head parameters are the same. 


\section{B. Half Pulse Width}

The half pulse width $P W_{50}$ at 50 percent of the voltage amplitude, is another non negligible physical quantity affecting the recording density. Since a maximal peak voltage gives the smaller half pulse width, the $P W_{50}$ plays also an important role in the manipulation of Linear Density (LD) in digital recording systems since the minimizing of this half pulse width implies the higher digital density [38]. This will give the opportunity to obtain and try as much as possible to improve the recording density of the recording medium due to this Arctan(exp)profile transition. Because obtainment or measurement of half pulse width using output voltage for isolated transition, gives much information for characterization of the recorded transitions and, digital recording density as we notice it previously. For example, if the signal pulse width at half maximum $\left(P W_{50}\right)$ is large, inter symbol interference will negatively impact the playback performance of the recording system. These results fall on to the predictions of Plummer et al [45], [46]. Otherwise, if $P W_{50}$ is small then the recording surface or the bit length is also small. Hence, the linear or areal density is high. For this reason, it is necessary to look for a half pulse width very small as much as possible. The half pulse width is defined as $P W_{50}$ $=2 Y_{50}$, where $V\left(y_{50}\right)=(1 / 2)$ Vpeak. This means that the half pulse width $Y_{50}$ is obtained involving the equation.

$\arctan \left(\frac{\cos \beta}{\sinh \left(\frac{y_{50}+g / 2}{\Delta}\right)}\right)+\arctan \left(\frac{\cos \beta}{\sinh \left(\frac{-y_{50}+g / 2}{\Delta}\right)}\right)=\arctan \left(\frac{\cos \beta}{\sinh \left(\frac{g}{2 \Delta}\right)}\right)-\frac{\pi}{2}$.

Now to obtain the half pulse width we should solve this equation with respect of $y_{50}$. Considering the fact, the solution of (29) is not far from $(-g / 2)$ this impliesthat the solution $y_{50}$ is closed to $(-g / 2)$. Hence the second terms of the right hand side of (29) is to small compare to the first one or tend to zero and then can be negligible term. At the end, equation becomes:

$\arctan \left(\frac{\cos \beta}{\sinh \left(\frac{y_{50}+g / 2}{\Delta}\right)}\right)=A$

where $A=\arctan \left(\frac{\cos \beta}{\sinh \left(\frac{g}{2 \Delta}\right)}\right)-\frac{\pi}{2}$

The analytical solution of (30) can be well done or analytically evaluated by considering the fact that $\tan A \simeq-\frac{\cos ^{2} \beta}{\sinh \left(\frac{g}{2 \Delta}\right)}$ and the half width can be done:

$$
\frac{Y_{50}}{\Delta}=\frac{g}{2 \Delta}-\ln \left[-\frac{\cos ^{2} \beta}{\sinh \left(\frac{g}{2 \Delta}\right)}+\sqrt{1+\left(\frac{\cos ^{2} \beta}{\sinh \left(\frac{g}{2 \Delta}\right)}\right)^{2}}\right]
$$

and the half pulse width is written given as:

$$
P W_{50}=2 Y_{50}=g-2 \Delta \ln \left[-\frac{\cos ^{2} \beta}{\sinh \left(\frac{g}{2 \Delta}\right)}+\sqrt{1+\left(\frac{\cos ^{2} \beta}{\sinh \left(\frac{g}{2 \Delta}\right)}\right)^{2}}\right]
$$

The $P W_{50}$ versus magnetic spacing $(d / \Delta)$ graph presented in Fig. 9 tell us that, in order to minimize half pulse width, the magnetic spacing $d / \Delta$ should be choosing so close to $(2 n+1) \frac{\pi}{2}$

. For this model Arctan(exp) function transition (DW) shape shows that our results are correct since good agreement is found with previous famous researcher works [47] and [48].

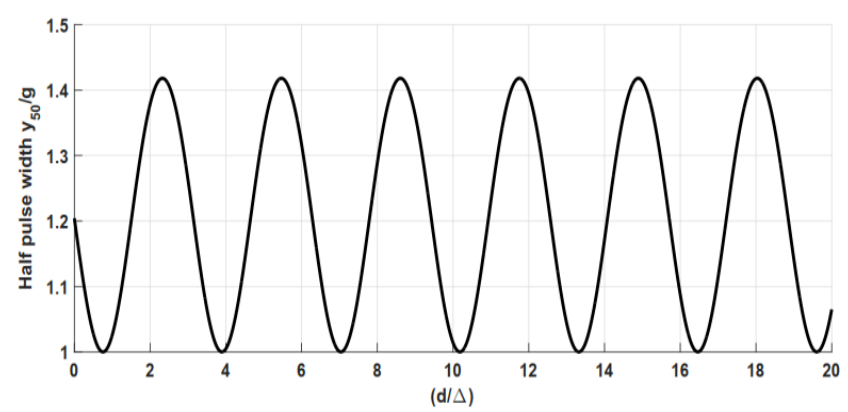

Fig. 9. Half pulse width $P W_{50}$ versus magnetic spacing $d / \Delta$ obtain with the $\operatorname{Arctan}(\exp )$ transition shape model.

This observation shows and proves that this model of transition shape is not far away from other model and has a little advantage. Then the model can be used for transition shape. Knowing that the half pulse width plays an important role in recording media, it is $P W_{50}$ which determines the behavior of linear recording density. The density can be defined as: the total number of information that can be stored in a unit surface area of media, this means that the smaller the size of information stored, the higher and the linear density. To have high digital recording density, we should have small size information to store and so, we need a very small half pulse width simply because it can be that $P W_{50}$ who determine the size of written or reading information in media. In addition, we realize by comparing $P W_{50}$ obtained with this model and the half pulse width obtain with tanh model in [48] that the slope of the graph $P W_{50}$ versus magnetic spacing $(d / \Delta)$ in the present Arctan model is smaller than in the tanh model and the same for other model of transition shape. Fig. 10 revealed the same observation. The comparison is made using results of [49].

This result informs us that the half pulse width in this Arctan(exp) model remains very small as the magnetic spacing increases since the progressively increasing density requires a progressively decreasing magnetic spacing between the head and data recording layer of disk media which is good when you have much information that can be written in the smaller area. We may conclude that with this 
transition shape, we can produce or recalled information of smaller size than when information is produced with tanh, old Arctan or other transition model.

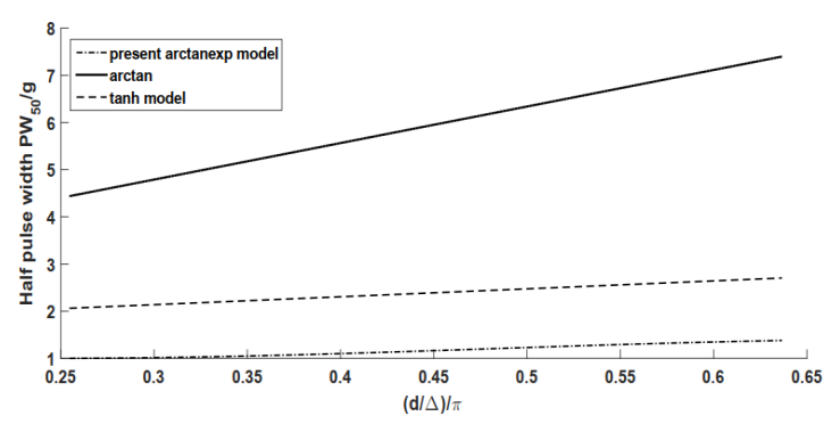

Fig.10. Comparison Half pulse width $P W_{50}$ versus magnetic spacing $d / \Delta$ graph of the Arctan(exp) transition shape model and two other shapes.

\section{DISCUSSION}

Domain wall is considered as one of the most important parameters in magnetic recording theory. This is emphasized in the new racetrack memory [24] using the propagation or the displacement of DW to stored and recalled information in the multilayer thin film. The shape, profile and DW width or transition length are elements which make it more attractive. Firstly, when the Domain Wall width decreases then the storage density increases, this reinforce the fact that DW are very important on the storage systems. In the present work the transition length relation $\Delta$ is propose (14), which derive from competition between the anisotropy and exchange energy. The relation is in concordance with previous work where we realize that transition length is determined by the combination of this two energy contribution [49]. The relation considers 3 different anisotropy direction $\left(K_{31}\right.$ and $K_{23}$ ) which is certainly good for practice when made storage media. Our result concerning DW width can be compare with another one [49], [50] where they proposed the DW width relation that has an uniaxial anisotropy $K u$, which is uncertain in real application and problematic when made the recording media because the new media are $3 D$ therefore to have that type of material with uniform anisotropy is more complicated. Hence to approaches the reality we should consider anisotropy in all anisotropic direction (1;2 and 3$)$ of the crystal as we did in this work to ensure the practicability of this Domain Wall types. Secondly, Domain Wall profile obtains in this present work, permit to conduct comparative study. In fact, many works have been done and they demonstrate that the DW can be model by the soliton form. Hence, several profiles (Arctan, tanh, erf) have been proposed and point up through some analysis. Considering the existence of many DW form, the choice of an adequate one becomes judicious in the aims of recording media performances and optimization. In this work we obtain KinkAntiKink Soliton profile (Arctan(exp)) (16) and elliptic solution meaningful Soliton Train by using equations states which governed the micro magnetic systems and through the Hamiltonian of magnetic spin chain. This transition shape put in highlight transition width which is a multiple of interatomic distance $a$. In the other transition shape, DW width are taken the same as the interatomic distance which is far away from the reality because we all know that for a real material the transition length is mostly greater and multiple of interatomic distance. This observation reinforces the validity and the uses of this $\operatorname{Arctan}(\exp )$ profile as the best one to describe the magnetic domain wall. The soliton train DW solution obtain here satisfy our expectation, in fact we realize that many DW putting together can exhibited the soliton train with good and well know period. This result is benefit when manipulating the new class of memory which needs to propagate DW by injection of current density or by applying external magnetic field. Apart type of materials with well choosing properties and domain wall profile there are many other parameters and measures than should be taken for achievement of high density in magnetic recording systems, where some of most important are: The gap length $g$ and the magnetic spacing $d$ or $d+2 \delta$. Concerning the magnetic spacing, we notice through this work that there is a particular relationships between the higher density and $d$. we observe that for high recording density, we should fine the smallest magnetic spacing because the smaller the spacing, the higher the peak voltage, the smaller the half pulse width and the higher the linear or areal density. This is in concordance with previous results which revealed that the necessary ingredient to high areal density is a close proximity of the write and read element of recording systems or the magnetic spacing has scaled with the length of the bit $(B)$ [51], [52]. Notice that we perform all our results using experimental values given by [53].

\section{CONCLUSION}

Domain walls have been used in several fields of physics but their role, importance and uses in magnetic recording systems is even more striking. In this paper, we have investigated and used the concept of soliton domain wall in magnetic recording systems. For this, from the magnetic media considered as a spin chain, we have through the Hamiltonian, certain interactions of first neighboring atoms (Spin), the Lagrangian of the system thus formed, we obtain one of Domain Wall (DW) profile (kink Soliton), and to some extent the soliton train. These results permit us to look feasibility with utility and if it is possible to use this domain wall (DW) transition shape in recording systems. For that, we have made a certain comparison of key parameters of recording systems such as: output voltage $V(y)$, peak voltage and half pulse width $\left(P W_{50}\right)$ with the same quantities of other transition shape. We conclude that with this transition shape, results are better and more accurate due to the fact that we perform most of our results using analytic methods, numerical simulation. Through the experimental values found in the literature, we were able to conduct this comparative study very well of the characteristic quantities of the system (Voltage, half pulse width, etc.) obtained with this soliton Kink profile and those obtained with other already existing profiles. The important verification that we should made is to ask if it is possible that this type of transition shape can be obtain with interaction between next nearest neighbor or within a frustrated system, investigate the uses of this profile contrary to others in the purpose of minimizing the effect of noises in recording systems and then, also look if possible, to use this DW shape in Current-Induced Domain Wall Motion (CIDWM). In other word, can this domain wall transition should be used in dynamic of magnetic domain wall 
with all other Micromagnetic effect? Using good analytical and mathematical methods in order to improve the Racetrack memories. The answers to these questions are new challenges for many researchers in magnetic recording systems since the Racetrack memory use DW propagation as key processes in this new class of recording system.

\section{ACKNOWLEDGMENT}

As research is not funded in our country, this work comes from our own funds. This work has not received financial support from our country or any other organization.

\section{REFERENCES}

[1] Parkin S. S. P, Hayashi M., and L. Thomas, "Magnetic domain-wall racetrack memory", Science (New York, N.Y.), 320 (5873): 190-4, April 2008.

[2] Fukami S, Suzuki T, Nagahara K, Ohshima N, Ozaki Y, Saito S, Nebashi R, Sakimura N, Honjo H, Mori K, et al, "Low-current perpendicular domain wall motion cell for scalable high speed MRAM ", InSymposium on VLSI Technology, 2009.

[3] Steven lequeux, Déplacement de paroi de domaine par transfert de spin dans des jonctions tunnel magnétiques : application au memristor spintronique, Thèse de doctorat de l'université paris-Saclay, préparée a l'université paris-sud école doctorale $n^{\circ} 564$ physique en Île-deFrance, spécialité: physique de la matière condensée, nanophysique, juin 2016.

[4] Chanthbouala A, Matsumoto R, Grollier J, Cros V, Anane A, Fert A, Khvalkovskiy A. V, Zvezdin K. A, Nishimura K, Nagamine Y, Maehara H, Tsunekawa K, Fukushima A, and Yuasa S, "Verticalcurrent-induced domain-wall motion in $\mathrm{MgO}$-based magnetic tunnel junctions with low current densities," Nature Physics, 7(8) :626-630, April 2011.

[5] Middelhoek S, "Domain Walls in Thin Ni [Single Bond] Fe Films," Journal of Applied Physics, 34, (4), 1054, June (1963).

[6] NEEL M. L, "Parois dans les films minces," Le Journal de Physique Colloque, 29(C2): C2-87-C2-94, February (1968).

[7] Thiaville A. and Nakatani Y, "Domain-Wall Dynamics in Nanowires and Nanostrips," Spin dynamics in confined magnetic structures III, 2006. URL http://link.springer.com/chapter/ 10.1007/10938171_5.

[8] MasanoriK, "Historical Development of Magnetic Recording and Tape Recorder," National Museum of Nature and Science Survey Reports on the Systemization of Technologies, vol.17, pp. 185-275, 2012.

[9] Kanellos M, "A divide over the future of hard drives," CNETNews.com, 2006.

[10] Robert H. Kraus, "the overview and history of permanent magnet devices in accelerator technology" Submitted to Thirteenth international conference on magnet technology, Victoria conference centre. Victoria, B. C. CANADA, September 20-24, 1993.

[11] Miles J.J and Middleton B.K, "An analysis for the magnetization mode for high density magnetic recording," IEEE Trans. Magn., vol. MAG23, No5, pp.2070-2072, 1987.

[12] Hoagland A. S. and Monson J. E, Digital magnetic recording, Editor: John Wiley \& Sons Inc; 2nd Revised edition (14 June 1991), PP 248. ISBN-10: 0471401447; ISBN-13: 978-0471401445.

[13] Smith D, Chunsheng E, Khizroev S, and Litvinov D, "The Influence of Bit Patterned Medium Design and Imperfections on Magnetoresistive Playback," IEEE Trans. Magn. 42, 2285, 2006.

[14] Bertram H. N, Theory of magnetic recording, Cambridge, University Press; 1994 PP 121.

[15] Middleton B. K, Recording and reproducing processes2nd Ed C.D. Mee, E.D. Daniel (Eds.), Magnetic Recording Handbook; 1996, Chap.3, pp. 82.

[16] IBM, "OEM MR Head Technology The erea of giant magnetoresistive heads," Hitachigst.com, Archived from the Retrieved 4 September 2010.

[17] Varvaco G and Casoli F," Ultrahigh density magnetic recording: Storage Materials and Media Designs," Pan Stanford Publishing Pte. Ltd;2016, pp. 457.

[18] H. N. Bertram, Theory of magnetic recording, Book in Cambridge University Press, 1994, pp 100.

[19] B. K. Middleton, Recording and reproducing processes, C. D. Mee, E. D. Daniel (Eds.), Magnetic Recording Handbook $2^{\text {nd }}$ Ed, 1996, Chap. 2, pp. 30 and Chap. 3, pp. 87.
[20] Freiser M. J, "On the zigzag form of charged domain walls," IBM J Res. Dev., vol. 23, pp. 330-338, May 1979.

[21] Muller M. and Murdock E, "Williams-Comstock type model for sawtooth transitions in thin film media," IEEE Trans. Magn., vol. 23, pp. 2368-2370, Sept. 1987.

[22] Minnaja N. and Nobile M, "Stability conditions for saw-tooth walls between head-on domains," AIP Conf. Proc. 10, 1001-5, 1972.

[23] Semenov V, Factorivich A. and Gikas M, "The effect of coercive squareness $\mathrm{S}^{*}$ on transition noise in thin metal media," Proc. Jap. Mag. Society of Japan 15 (S2), 251-6, 1991.

[24] Parkin S. S. P, Hayashi M and Thomas L, "Magnetic Domain-Wall Racetrack Memory,"Science, vol.320, pp. 190, 2008.

[25] Mallinson J. C, "On extremely high density digital recording," IEEE Transaction on Magnetics Mag, vol. 10, No.2, 1974.

[26] Murali K. H, "Time resolved domain wall motion in novel spin transfer torque devices,"PhD thesis, Department of Physic, University of Bath, Bath, UK, 2015.

[27] Hozoi A, "Edge effects and submicron track in magnetic tape recording,"PhD thesis, University of Twente, Romania, 2005.

[28] Thiaville A, Rohart S, Jué E, Cros V and Fert A, "Dynamics of Dzyaloshinskii domain walls in ultrathin magnetic films," Europhysics Lett, vol. 100, pp. 57002, 2012.

[29] Galkina E. G, Ivanov B A, Savelev S and Nori F, "Chirality tunneling and quantum dynamics for domain walls in mesoscopic ferromagnets," Physical reviews B, vol. 77, pp. 34425, 2008.

[30] Takagi S and Tatara G, "Macroscopic quantum coherence of chirality of domain wall in ferromagnets," Physical reviews B, vol. 54, Num.14, 1996.

[31] Galkina E. G, Ivanov B. A, Savelév S and Nori Franco, "Chirality tunneling and quantum dynamics for domain walls in mesoscopic ferromagnets," PHYSICAL REVIEW B, vol.77, pp. 134425, 2008.

[32] Malozemoff A P and Slonczewski J C, Magnetic domain walls in bubble materials, New York: Academic Press; 1979, pp 78.

[33] Mathieu Cormier, "Renversement d'aimantation dans des nanostructures par propagation de parois de domaines sous champ magnétique et courant électrique,"Thèse de Doctorat en Physique, Université Paris-Sud 11, France, 2008, pp. 29.

[34] Michel Remoissenet, Waves called solitons: concepts and experiments, 2nd edition, Berlin Heidelberg: Springer-Verlag;1994.

[35] Yemélé D and kofané T. C, "Long-range effect on the periodic deformable Sine-Gordon chains," Journal of Physics: Condensed Matter, vol.11, Issue 11, pp. L75-L81, 1999.

[36] Webster J, Wiley Encyclopedia of Electrical and Electronics Engineering, John Wiley \&Sons, Inc; 1999.

[37] Morrish A. H, The physical principles of magnetism, New York: Wiley; 1965.

[38] Potter R. I,"Digital magnetic recording theory," IBM Thomas J. Watson research center, Yorktown heights, New York,10598, 1974

[39] H. Neal Bertram, Hong Zhou and Roy Gustafson, "Signal to noise ratio scaling and density limit estimates in longitudinal magnetic recording," IEEE Trans on Magn, vol. 34, No, 4, July 1998.

[40] Bertram H. N and Che X, "General analysis of noise in recorded transitions in thin film recording media," IEEE Trans on Magn, vol. 29 No.1, 1993.

[41] Slutsky B and Bertram H. N, "Transition noise analysis of thin film magnetic recording media," IEEE Trans on Magn, vol. 30, No.5, 1994

[42] Karlqvist, "Calculation of the magnetic field in the ferromagnetic layer of a magnetic drum," Trans. R. Inst. Technol Stockholm, vol. 86 pp. 3 , 1954.

[43] Bertram H. N, Theory of magnetic recording, Cambridge, University Press; 1994, pp. 100.

[44] Middleton B. K, Recording and reproducing processes2nd Ed C.D. Mee, E.D. Daniel (Eds.), Magnetic Recording Handbook;1996, Chap. 2, pp. 30 and Chap.3, pp. 87.

[45] Aziz M. M, Signal and Noise Properties of Longitudinal Thin Film Disk Media, thesis Faculty of Science and Engineering, University of Manchester, UK, 1999, Chap. 3 pp. 86-88.

[46] Plumer M. L, van J. E and Weller D, The physics of ultra-high-density magnetic recording, Springer Series in Surface Sciences,2001, PP. 2627.

[47] Zhan Y and Bertram H. N, "PW50, D50 and playback voltage formulas for shield magnetoresistive heads," Journal of Applied Physics, vol.81, pp. 4897, 1997.

[48] Zhan Y, Shtrikam S and Bertram H. N, "Playback pulse shape and spectra for shielded MR heads, " IEEE Trans on Magn, vol. 33, No. 2, 1997.

[49] Wang L and Han G,"Down track response of differential reader for high density magnetic recording" Data Storage Institute, Agency for Science, Technology and Research (A*STAR), 2011. 
[50] Kittel C, "Physical theory of ferromagnetic domains, "Reviews of modern physics, vol. 21, Number 4, 1949.

[51] Mougin A, Cormier M, Adam J. P, Metaxas P J and Ferré J, "Domain wall mobility stability and Walker breakdown in magnetic nanowires," Europhysics Letters (EPL), vol. 78, pp. 57007, 2007.

[52] Marchon B. and Olson Terry, "Magnetic Spacing Trends: From LMR to PMR and Beyond," IEEE TRANSACTIONS ON MAGNETICS, vol. 45, No.10, 2009.

[53] Middleton B. K, Miles J. J and Aziz M.M, "High-density recording on magnetic thin film disk media," Journal of Magnetism and Magnetic Materials, vol. 188, pp.248-259, 1998.

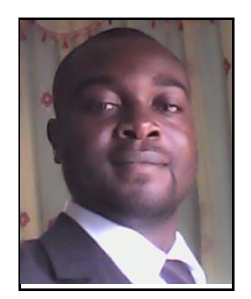

Tefouet Noula J. Duclair was born September 03, 1984 in Foumbot in the West region of Cameroon. Holder of a Master of Science, he is actually $\mathrm{PhD}$ student and doing his research works in the field of "theoretical and numerical analysis of magnetic recording systems" within the Research Unit of Mechanics and Modeling of Physical Systems of the Faculty of Sciences, University of Dschang (Cameroon).

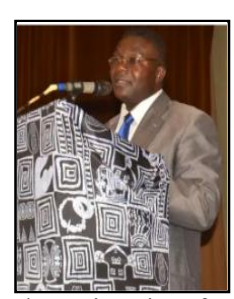

Prof. Yemélé David is a teacher and researcher at the University of Dschang (Cameroon). Full Professor of Universities, he currently directs work in several fields of nonlinear physics, analysis of structures, signal processing and renewable energies within the research unit of mechanics and modeling of physical systems of the faculty of sciences from the University of Dschang and the Mechanics, Materials and Structures Laboratory of the University of Yaoundé 1 (Cameroon). 